\title{
Overview of the Recent DiMES and MiMES Experiments in DIII-D
}

D.L. Rudakov ${ }^{\mathrm{a}, *}$, C.P.C Wong ${ }^{\mathrm{b}}$, A. Litnovsky ${ }^{\mathrm{c}}$, W.R. Wampler ${ }^{\mathrm{d}}$, J.A. Boedo ${ }^{\mathrm{a}}$,

N.H. Brooks ${ }^{\mathrm{b}}$, M.E. Fenstermacher ${ }^{\mathrm{e}}$, M. Groth ${ }^{\mathrm{e}}$, E.M. Hollmann ${ }^{\mathrm{a}}$, W. Jacob ${ }^{\mathrm{f}}$, S.I. Krasheninnikov ${ }^{\mathrm{a}}$, K. Krieger ${ }^{\mathrm{f}}$, C.J. Lasnier ${ }^{\mathrm{e}}$, A.W. Leonard ${ }^{\mathrm{b}}$, A.G. McLean ${ }^{\mathrm{g}}$, M. Marot ${ }^{\mathrm{h}}$, R.A. Moyer ${ }^{\mathrm{a}}$, T.W. Petrie ${ }^{\mathrm{b}}$, V. Philipps ${ }^{\mathrm{c}}$, R.D. Smirnov ${ }^{\mathrm{a}}$, P.C. Stangeby ${ }^{\mathrm{g}}$, J.G. Watkins ${ }^{\mathrm{d}}$, W.P. West ${ }^{\mathrm{b}}$, and J.H. Yu ${ }^{\mathrm{a}}$

${ }^{a}$ University of California, San Diego, La Jolla, California 92093-0417, USA

${ }^{b}$ General Atomics, P.O. Box 85608, San Diego, California 92186-5608, USA

${ }^{c}$ Forschungszentrum Jülich, EURATOM Association, 52425, Jülich, Germany

${ }^{d}$ Sandia National Laboratories, Albuquerque, New Mexico 87185, USA

${ }^{e}$ Lawrence Livermore National Laboratory, Livermore, California 94551, USA

${ }^{f}$ Max-Planck-Institut für Plasmaphysik, EURATOM Association, 85748 Garching, Germany

${ }^{g}$ University of Toronto Institute for Aerospace Studies, Toronto, M3H 5T6, Canada.

${ }^{h}$ University of Basel, Klingelbergstrasse 82, CH-4056 Basel, Switzerland.

*E-mail: rudakov@fusion.gat.com

\begin{abstract}
Divertor and midplane material evaluation systems (DiMES and MiMES) in the DIII-D tokamak are used to address a variety of plasma-material interaction issues relevant to ITER. Among the topics studied are carbon erosion and re-deposition, hydrogenic retention in the gaps between plasma facing components (PFCs), deterioration of diagnostic mirrors from carbon deposition and techniques to mitigate that deposition, and dynamics and transport of dust. An overview of the recent experimental results is presented.
\end{abstract}

PACS: 52.40.Hf, 52.25.Vy, 52.55.Fa 


\section{Introduction}

Maintaining plasma-material interactions (PMI) at an acceptable level is a serious challenge for the next generation of fusion devices, such as ITER [1]. Particle and energy fluxes incident on the plasma-facing components (PFCs) will have fluencies orders of magnitude higher than those in contemporary machines, resulting in much higher erosion than presently observed. The current ITER design incorporates beryllium tile cladding of the main chamber walls and a divertor with tungsten baffles and carbon fibre composite (CFC) target plates [2]. Carbon will erode from the plates and redeposit elsewhere leading to potential problems such as co-deposition of tritium and formation of dust. In addition, transient events such as edge-localized modes (ELMs) and disruptions may result in enhanced erosion and even localized melting of the metallic PFCs, contributing to the formation of dust and re-deposited layers containing mixed materials and tritium. Inventories of both tritium and dust in ITER are strictly regulated, so projections of accumulation rates as well as development of accumulation mitigation and removal techniques are needed. In addition, impurities resulting from PFC erosion and dust can penetrate the core plasma and degrade performance; this has to be avoided. Another problem related to erosion/re-deposition is deterioration of diagnostic mirrors that will be used by a majority of ITER optical diagnostics as a plasma-viewing element [3]. Deposition of carbon and/or beryllium on the mirrors may shut down the diagnostics working in the ultraviolet and visible wavelength ranges. Deposition mitigation and mirror cleaning techniques need to be developed to prevent this from occurring. 


\section{DiMES and MiMES Systems in DIII-D}

DIII-D [4] is a tokamak with major and minor radii of $1.67 \mathrm{~m}$ and $0.67 \mathrm{~m}$, respectively. It has two poloidal divertors at the top and bottom of the vacuum vessel and can be operated in lower single null (LSN), upper single null (USN), double null (DN) and wall-limited magnetic configurations. Divertor and main chamber wall surfaces are lined with graphite tiles. The lower divertor is equipped with the Divertor Material Evaluation System (DiMES) [5,6] allowing insertion of material samples flush with the divertor floor tiles. In addition, Midplane Material Evaluation System (MiMES) [6] allowing exposure of samples just inside of the wall tile radius near the outboard midplane has been recently commissioned. This system is combined with the mid-plane reciprocating probe (RCP) providing measurements of the plasma density and temperature profiles in the outboard scrape-off layer (SOL). Figure 1(a) shows a photograph of MiMES inside DIII-D vacuum vessel with RCP fast stroke in inserted position. Locations of 4 material "button” samples at the plasma-facing side are marked by small arrows. Larger arrows show positions of the mirror sample slots at the bottom and side surfaces. A photograph of MiMES with two mirrors installed is shown in Fig. 1(b). 


\section{Studies of Carbon Erosion and Re-deposition}

Previous studies of carbon erosion/re-deposition in the lower divertor of DIII-D using DiMES typically showed net erosion near the outer strike point (OSP) in discharges with an attached OSP and tendency towards net deposition in discharges with a detached OSP [7]. However, a very high erosion rate $(\sim 15 \mathrm{~nm} / \mathrm{s})$ was observed at the OSP in high confinement (H-mode) plasmas detached by neon injection [8]. This high erosion rate was explained by chemically enhanced physical sputtering by neon. However, during that experiment the divertor plasma oscillated between a predominant low electron temperature $\left(T_{\mathrm{e}}=1.5-4 \mathrm{eV}\right.$ ) high density state and a less frequent higher temperature $\left(T_{\mathrm{e}}=12-30 \mathrm{eV}\right)$ lower density state, which may have influenced the result.

Experiments with argon injection were recently performed in order to see whether the high erosion observed with neon would reproduce. Due to some operational constraints, full OSP detachment was not achieved, and the DiMES sample was exposed to two ELMing H-mode discharges (discharges \#130005 and 130007) with neutral beam injection (NBI) heating power of $7 \mathrm{MW}$ for a total of $\sim 7$ plasma seconds with the OSP attached for the majority of the exposure ( $6 \mathrm{~s}$ total duration), and detaching near the end of the discharges ( $1 \mathrm{~s}$ total). The sample made of ATJ graphite had implanted silicon depth marker allowing measurements of the net erosion/deposition by ion beam analysis (IBA). Tungsten and vanadium stripes $\sim 100 \mathrm{~nm}$ thick and $3 \times 30 \mathrm{~mm}^{2}$ in size were deposited on the sample surface to examine the erosion of metals [Fig. 2(a)]. Results of IBA analysis of the sample are presented in Fig. 2(b) (negative values correspond to erosion). The OSP location during the exposure is shown by a shaded area accounting for a slight OSP motion and error margins of the EFIT magnetic reconstruction [9]. Peak net 
erosion of $\sim 60 \mathrm{~nm}$, corresponding to erosion rate of $\sim 9 \mathrm{~nm} / \mathrm{s}$ during the plasma exposure was observed near the OSP. Erosion of tungsten from the deposited stripe and redeposition on the adjacent graphite surface were also observed [Fig. 2(a)]. The fact that the carbon erosion rate in this experiment was $40 \%$ lower than that reported in [8], even though OSP was attached most of the time, suggest that oscillations between attached and detached state did affect the results of Ref. [8].

Two exposures of DiMES samples to ELMing H-mode discharges with NBI heating power of 6.8 MW and OSP detached by neon injection have been recently performed. Each exposure was to 3 plasma discharges, the first one for a total of $\sim 9$ plasma seconds (discharges \#136820-22) and the second one for 10.5 seconds (discharges \#136916-18). Seven "button" samples $\sim 6 \mathrm{~mm}$ in diameter were exposed in both cases. Samples included depth-marked graphite (two samples per exposure), diamond-coated silicon, tungsten and blank graphite buttons [Fig. 3]. The first exposure was performed with the samples kept at the ambient temperature, and the second one was with the samples preheated to $300^{\circ} \mathrm{C}$ using an electrical heater built into the sample [10]. Upon extraction of the sample following the first (non-heated) exposure, visible deposits were found on the plasma-facing surface of the button holder [Fig. 3]. No deposits were observed after the second (heated) exposure. IBA analysis of the depth-marked graphite buttons has been performed at Sandia National Laboratories, Albuquerque. Both buttons exposed at the ambient temperature showed bias towards net deposition, though the deposit thickness was too small to be resolved. The buttons exposed at $300^{\circ} \mathrm{C}$, on the contrary, showed very high net erosion. The button located during the exposure just inside of the OSP (position A in Fig. 3) had net erosion of $230 \pm 10 \mathrm{~nm}$, corresponding to erosion rate of $\sim 22 \mathrm{~nm} / \mathrm{s}$. 
The button located just outside of the OSP (position B in Fig. 3) had erosion in excess of $330 \mathrm{~nm}$ (the exact number could not be determined since the implanted depth marker eroded away), corresponding to erosion rate of above $31 \mathrm{~nm} / \mathrm{s}$. These results are in qualitative agreement with previous DiMES experiments which showed a strong dependence of the carbon erosion rate on the surface temperature under the detached divertor conditions [11]. However, the erosion rate measured in the latest experiment was an order of magnitude higher than that reported in [11] at $200^{\circ} \mathrm{C}(\sim 3 \mathrm{~nm} / \mathrm{s})$. Whether the erosion enhancement was due to the presence of neon, higher surface temperature $\left(300^{\circ} \mathrm{C}\right.$ versus $200^{\circ} \mathrm{C}$ ) or difference in the plasma conditions (H- versus L-mode) remains to be investigated.

First measurements of the net erosion/deposition near the outboard main chamber wall have recently been performed using MiMES [12]. Depth-marked button samples were installed on the plasma-facing side of MiMES (slots 1 and 4 in Fig. 1(a)) and exposed in two series of ELMing H-mode discharges. The first series of 7 discharges (\#132772-78) with NBI heating power of 5 MW was high-density (electron density normalized to Greenwald limit, $n_{\mathrm{e}} / n_{\mathrm{GW}} \sim 1$ ) with small ELMs. The second series of 10 discharges (\#134266-75) with NBI heating power of $\sim 4$ MW was of lower density $\left(n_{\mathrm{e}} / n_{\mathrm{GW}} \sim 0.45\right)$ with larger ELMs. The discharges had similar equilibrium shape and stored pedestal energy. More experimental details are available in [12]. Net carbon deposition of $24 \mathrm{~nm}$ and $40 \mathrm{~nm}$ was measured on the two samples exposed for a total of 28 plasma seconds in the high-density discharge series (with the measurement uncertainty of $\pm 10 \mathrm{~nm}$ ). Judging by this result, in high density conditions with small ELMs the outboard main chamber PFCs are not subject to any significant erosion, and 
may even be subject to net deposition. Analysis of the two samples exposed in the lower density discharge series for a total of $\sim 40$ plasma seconds showed net erosion of $13 \mathrm{~nm}$ and $10 \mathrm{~nm}$. While barely out of the uncertainty of the measurement, this result suggests that in lower density regimes with larger ELMs net erosion of the outboard main chamber PFCs is more likely to occur. 


\section{Hydrogenic Retention in PFC Gaps}

PFCs in ITER will be castellated to reduce the thermal stress. Fuel retention in the castellation gaps represents a potential safety issue and has to be mitigated. Two different mitigation techniques have been recently tested in DIII-D, one involving an elevated surface temperature and another one using shaped castellations.

Previous DiMES experiments used a sample with a gap $2 \mathrm{~mm}$ wide and $18 \mathrm{~mm}$ deep lined with silicon catcher plates [11,13]. A factor of 3-4 reduction of carbon deposition rate and an order of magnitude reduction of the deuterium co-deposition rate in the gap was achieved by increasing the sample temperature from $\sim 30^{\circ} \mathrm{C}$ to $\sim 200^{\circ} \mathrm{C}$ $[11,13]$. Recently, another exposure of the tile gap sample pre-heated to between $200^{\circ}-$ $300^{\circ} \mathrm{C}$ was performed in 7 L-mode LSN discharges (\#134196-202) with the divertor plasma parameters comparable to those in the previous exposures. Unlike in the previous experiments, the catcher plates were made of copper to eliminate the possibility of carbon reacting with the plate material at high temperatures. Further reduction of $\mathrm{D}$ codeposition rate compared to the previous heated exposure was observed. Deuterium areal density on the catcher plates near the gap entrance, as measured by Nuclear Reaction Analysis, was $3 \times 10^{15}( \pm 25 \%)$ atoms $/ \mathrm{cm}^{2}$ at the gap side facing the incident plasma flux and $1.7 \times 10^{15}( \pm 25 \%)$ atoms $/ \mathrm{cm}^{2}$ at the shaded side. For comparison, D density near the gap entrance at the shaded side of the gap after the previous heated exposure was $\sim 4 \times 10^{15}$ atoms $/ \mathrm{cm}^{2}$ (see Fig. 2(b) in Ref. [11]).

Shaping of castellation cells is another way to reduce impurity and fuel accumulation in the gaps. First studies of the impact of castellation shaping in TEXTOR [14] showed a significant reduction of the fuel inventory in the shaped cells, accompanied with only a 
marginal reduction of the carbon transport. To investigate an impact of castellation shaping under fluxes incident at shallow angles, a special DiMES tungsten castellated sample was manufactured (Fig. 4). Rectangular castellation blocks [\#1-4 in Fig. 4(a)] were mounted in a graphite enclosure flush with the divertor tile surface, along with a strongly shaped block similar to those exposed in TEXTOR [\#6 in Fig. 4] and a block of optimized (less pronounced) shaping [\#5 in Fig. 4)] to allow a direct comparison between the three geometries. Shape optimization was required to minimize the plasma-shadowed areas believed to be responsible for the enhanced carbon accumulation in the shaped gaps.

The castellated DiMES sample was exposed in a series of ten identical LSN ELMy H-mode discharges (\#134248-57) with a partially detached OSP and NBI heating power of $5 \mathrm{MW}$ for a total of $\sim 45$ plasma seconds. During the exposure, the castellation was located in the private flux region and kept at the ambient temperature. After the exposure, thin stripe-like deposition patterns were formed on the side walls of the gaps [Fig. 4(c)]. Analyses of the deposition patterns were made with electron-probe micro-analysis technique (EPMA). An intensity of $K \alpha$ emission from an inner-shell electron of a carbon atom was used to estimate the absolute areal carbon concentration and its e-folding length in the downward direction on the poloidal side walls of the gaps. On the sides of all blocks (rectangular, strongly and moderately shaped) facing the plasma flux (incident along the toroidal magnetic field, $B_{T}$, in Fig 4(a)), the e-folding length, $\lambda_{\text {ec }}$, was between $0.4-0.6 \mathrm{~mm}$ (the measurement accuracy was rather poor due to the deposition thickness being much smaller than the surface roughness). At the same time, there was a significant difference in the carbon deposition patterns measured on the sides of the blocks 
shadowed from the incident flux: on the plasma-shadowed side of the strongly shaped cell (right side of block \#5 in Fig. 4(b)) $\lambda_{\text {ec }}$ was around $1 \mathrm{~mm}$, whereas on the plasmashadowed side of the moderately shaped cell (right side of block \#4 in Fig. 4(b)) and on the corresponding side of the rectangular cell (\#1) $\lambda_{\text {ec }}$ was $\sim 0.25 \mathrm{~mm}$. The integral amount of carbon accumulated in the poloidal gaps between rectangular blocks was $1.1 \times 10^{17}$ atoms (between blocks \#1 and \#2) and $1.7 \times 10^{17}$ atoms (between blocks \#2 and \#3). The amount of carbon in the strongly shaped gap (between blocks \#5 and \#6) was comparable at $1.6 \times 10^{17}$ atoms, while the amount of carbon in the moderately shaped gap (between blocks \#4 and \#5) was the lowest at $8.6 \times 10^{16}$ atoms, showing advantages of shaping optimization in the reduction of carbon deposition in the gaps. However, we should not over-interpret these results, since the deposit thickness was very small. A longer-term exposure of a similar castellated sample was recently performed in DIII-D. The results are still in the process of analysis and will be reported elsewhere. 


\section{Mirror Studies}

Previous experiments with diagnostic mirrors in DiMES demonstrated a strong reduction of the carbon deposition on the mirrors at an elevated surface temperature [10,15], which was also in line with the findings from ASDEX Upgrade [16]. An alternative mitigation approach involves a local deuterium gas injection in the vicinity of the mirrors. Following a successful application of this "gas puffing” technique to achieve active control over deposition in TEXTOR [17], a dedicated experiment was performed with diagnostic mirrors in the divertor of DIII-D. Two polycrystalline molybdenum mirrors were installed on the DiMES mirror holder $[10,15]$ and exposed in the private flux region of the lower divertor of DIII-D to seven identical NBI-heated high-density ( $\left.n_{\mathrm{e}} / n_{\mathrm{GW}} \sim 1\right) \mathrm{H}$-mode discharges (\#132772-78). Massive puffing of the deuterium gas $\left(D_{2}\right)$ at a rate of 71 Torr $\cdot 1 /$ s was provided during the exposure discharges using a capillary system located $12 \mathrm{~cm}$ upstream from the DiMES position at the same radial location. During the exposure mirrors were kept at the ambient temperature.

Optical characterization and surface analyses of the mirrors were performed before and after the exposure in DIII-D. A significant reduction of deposition was observed on the mirrors exposed with gas puff compared to those exposed without gas puff under similar plasma conditions and for nearly the same duration [15], as illustrated in Fig. 5(a).

Secondary ion mass spectrometry (SIMS) investigations of the elemental composition of the surface layer along with a depth profiling of the exposed mirrors were made using ION-TOF facility. A $2 \mathrm{keV}$ beam of Cs+ ions was used as a sputter beam for analysis of the deposits. Comparative analysis of the carbon content on the surface of the mirrors is shown in Fig. 5(b) (low signal intensity near the surface may be caused by surface effects 
and/or instability of the analyzing Bi-beam current). About a ten-fold reduction of the carbon layer thickness was inferred from the SIMS measurements performed on the mirrors exposed with the gas puff as compared to those exposed without the gas puff. Therefore, gas puff proved to be an efficient technique for an active control over deposition. The physical mechanisms that may contribute to the reduction of the carbon deposition rate on the mirrors include the decrease of the local carbon concentration in the vicinity of the mirrors due to the increased shielding by $\mathrm{D}_{2}$ gas (collisional damping) and binding of $\mathrm{C}$ atoms by $\mathrm{D}$ atoms resulting from $\mathrm{D}_{2}$ molecule dissociation (chemical damping), as well as increased chemical re-erosion by D ions and neutrals. However, the deposition on the mirrors exposed with the gas puff still caused a significant drop in reflectivity which would be detrimental to diagnostic sensitivity, calling for further optimization of the technique.

The first exposure of diagnostic mirrors under the main chamber conditions was performed using MiMES. Two mirrors were installed on MiMES as illustrated in Fig. 1(b) and exposed to 22 high density L-mode discharges (\#130114-35) for a total of about 70 plasma seconds. A rhodium-coated molybdenum mirror was oriented in a vertical plane (slot A) and located inside the port to have a toroidal view. A single-crystal molybdenum mirror was oriented in a horizontal plane (slot B) and located in the SOL just inside of the wall tile radius to have a view of the lower divertor. Both mirrors were shielded from the ionic flux, mirror in slot B because of its orientation and mirror in slot A because it was recessed 3-4 cm inside the port, where the plasma density is negligible. Upon extraction, ellipsometry and reflectivity analyses were performed on the mirrors. Ellipsometry revealed a carbon layer 5-10 nm thick on the toroidally viewing mirror. The 
total reflectivity of that mirror decreased by $10-15 \%$ in the wavelength range between 200-400 nm, while the reflectivity of the mirror viewing the divertor decreased by less than $5 \%$. Since the mirror that was $\sim 5 \mathrm{~cm}$ further away from the plasma received more deposition, it may be concluded that the deposition rate has a strong dependence on the mirror orientation. 


\section{Injections of Pre-characterized Dust for Studies of Dust Dynamics and Transport}

Injections of pre-characterized dust from a known location may be used to calibrate diagnostic measurements and benchmark modeling of dust dynamics and transport. Migration of carbon dust has been previously studied in DIII-D by introduction of micron-size graphite dust in the lower divertor [18]. Typically, 25-40 mg of dust was placed on a graphite DiMES sample holder and exposed to ELMing H-mode discharges with strike points swept across the divertor floor. Following a brief exposure $(\sim 0.1 \mathrm{~s})$ at the OSP, part of the dust was injected into the plasma, raising the core carbon density by a factor of 2-4 and resulting in up to a twofold increase of the total radiated power. Following the dust injection, individual dust particles were observed moving at velocities of $10-100 \mathrm{~m} / \mathrm{s}$, with trajectories elongated in the toroidal direction, consistent with acceleration by the ion drag force. The UCSD fast-framing camera (Phantom 7.1 [19]) observed large amounts of injected dust in the outboard SOL 90 deg or more toroidally away from the injection point [20]. Observed trajectories of dust particles in DIII-D are in qualitative agreement with modeling by the Dust $T$ code [21,22]. The range of the dust velocities predicted by the code is in good agreement with the camera data.

In the most recent injection experiment, spherical graphite dust (manufactured by Tokai Carbon Co, Ltd) with an 8 micron median diameter was used. The DiMES sample holder was loaded with $~ 30 \mathrm{mg}$ of the dust mixed with ethanol and allowed to dry forming a uniform layer. Afterwards, 10 mg of loose dust was placed on top of the dried layer. The first discharge with DiMES inserted disrupted 90 ms from the beginning. Starting at 13 ms into the discharge, large amounts of glowing dust were observed by the 
fast-framing camera running at 2000 frames/s and looking tangentially away from DiMES [Fig. 6]. The dust presumably prevented normal development of the discharge and caused the disruption. The experiment was later repeated with a similar result. Dust particle velocities inferred from the fast camera data were rather low, about $10 \mathrm{~m} / \mathrm{s}$ or less. Due to its low velocity, the dust could not travel a few meters from DiMES into the camera field of view in $13 \mathrm{~ms}$. The dust, therefore, must have become mobile and spread around the vacuum vessel prior to the discharge. This was confirmed by the Thomson scattering diagnostic data showing high level of scattered signal starting at $300 \mathrm{~ms}$ before the discharge. Possible mechanisms that could contribute to the mobilization and spreading of the dust are gas pre-fill, magnetic field ramp-up (graphite is diamagnetic), and electric field ramp-up for plasma breakdown. The electric field is the most likely candidate, since in laboratory tests with a Tesla coil the spherical graphite dust used in the experiment was observed to charge up and became mobile. While the results of this experiment may be specific to this particular type of dust, it has been previously shown that tritiated dust can levitate in electric field [23], so in a device like ITER operating with a D-T plasma, dust mobilization prior to a discharge may be a potential problem. 


\section{Discussion and Summary}

Recent experiments conducted in DIII-D using DiMES and MiMES have addressed a number of critical ITER-relevant PMI issues. A high erosion rate of graphite was observed in the divertor under semi-detached conditions with argon injection and under detached conditions with neon injection at elevated surface temperature. Care should be taken to avoid this problem if argon or neon injection is used to induce detachment in ITER. Reduction of the hydrogenic retention in PFC gaps at elevated temperature has been confirmed, which may be positive for ITER where PFC temperature will be higher than in most contemporary machines. The effect of the castellation shaping on fuel retention in gaps has also been demonstrated; however, more research is needed to optimize the shape for different conditions. Encouraging results have been obtained with D gas puffed over diagnostic mirrors resulting in a significant reduction of carbon deposition on the mirrors. Mirror exposure in the main chamber demonstrated a strong dependence of carbon deposition on the mirror orientation. Small amounts of dust introduced in DIII-D caused a rather strong effect on the plasma discharges. In particular, the latest experiment with spherical graphite dust showed that dust can mobilize prior to a discharge and prevent a normal discharge startup. This may be a potential problem in ITER, where significant amounts of dust are expected to be present on plasma-facing surfaces, and tritiated dust may be mobilized by electric fields. 


\section{Acknowledgments}

This work was supported in part by the US DOE under DE-FG02-07ER54917, DEFC02-04ER54698, and DE-AC52-07NA27344. Sandia is a multi-program laboratory operated by Lockheed Martin Company, for the United States Department of Energy’s National Nuclear Security Administration under contract DE-AC04-94AL85000. 


\section{References}

[1] Federici G et al 2001 Nucl. Fusion 411967

[2] Federici G et al 2008 Phys. Scr. T124 1

[3] Litnovsky A et al 2007 Nucl. Fusion 47833

[4] Luxon J L 2002 Nucl. Fusion 42614

[5] Wong C P C et al 1998 J. Nucl. Mater. 258-263 433

[6] Wong C P C et al 2007 J. Nucl. Mater. 363-365 276

[7] Whyte D G et al 2001 Nucl. Fusion 411243

[8] Wampler W R et al 2003 J. Nucl. Mater. 313-316 333

[9] Lao L L et al 1985 Nucl. Fusion 251611

[10] Rudakov D L et al 2006 Rev. Sci. Instrum. 77 10F126

[11] Rudakov D L et al 2007 Phys. Scr. T128 29

[12] Rudakov D L et al 2009 J. Nucl. Matter. 390-391 785

[13] Krieger K et al 2007 J. Nucl. Mater. 363-365 870

[14] Litnovsky A et al 2009 J. Nucl. Mater. 386-388 809

[15] Litnovsky A et al 2008 Fusion Eng. Des. 8379

[16] Mayer M et al 2006 Nucl.Fusion 46914

[17] Litnovsky A et al 2009 Nucl. Fusion 49075014

[18] Rudakov D L et al 2007 J. Nucl. Mater. 363-365 227

[19] Yu J H et al 2008 Phys. Plasmas 15032504

[20] Rudakov D L et al 2008 Rev. Sci. Instrum. 7910 F303

[21] Pigarov AYu et al. 2005 Phys. Plasmas 12122508

[22] Smirnov R D et al. 2007 Plasma Phys. Control. Fusion 49347

[23] Skinner C H et al. 2003 Fusion Sci. Tecnol. 4511 


\section{Figure captions}

Fig. 1: (a) MiMES inside DIII-D vacuum vessel; small arrows show the locations of material "button" samples, larger arrows show the positions of the mirror sample slots. (b) MiMES with mirrors installed.

Fig. 2: (a) photograph of the depth-marked DiMES sample exposed to plasmas with argon injection; (b) radial profile of the net carbon erosion/deposition measured by IBA along the line marked in (a). Direction of the toroidal magnetic field, $B_{T}$, during the exposure is shown in (a).

Fig. 3: DiMES head with multiple "button” samples after exposure to detached plasmas with neon injection.

Fig. 4: (a) tungsten castellation in DiMES graphite housing; (b) side view of the castellation removed from the housing; (c) flux-facing poloidal gap surfaces of blocks \#2 and \#5 after exposure in DIII-D.

Fig. 5: Mitigation of carbon deposition caused by deuterium gas puffing in the vicinity of diagnostic molybdenum mirrors exposed in the divertor of DIII-D: (a) photographs of the mirrors exposed without gas puff [15] and with gas puff; (b) results of SIMS depth profiling of these mirrors: solid symbols with gas puff, open symbols without gas puff. Measurement locations are shown in (a).

Fig. 6: Injected spherical carbon dust observed by a fast-framing camera $15 \mathrm{~ms}$ (b) and $26 \mathrm{~ms}$ (c) after the beginning of the discharge that ended in early disruption. The camera field of view is shown in (a). 

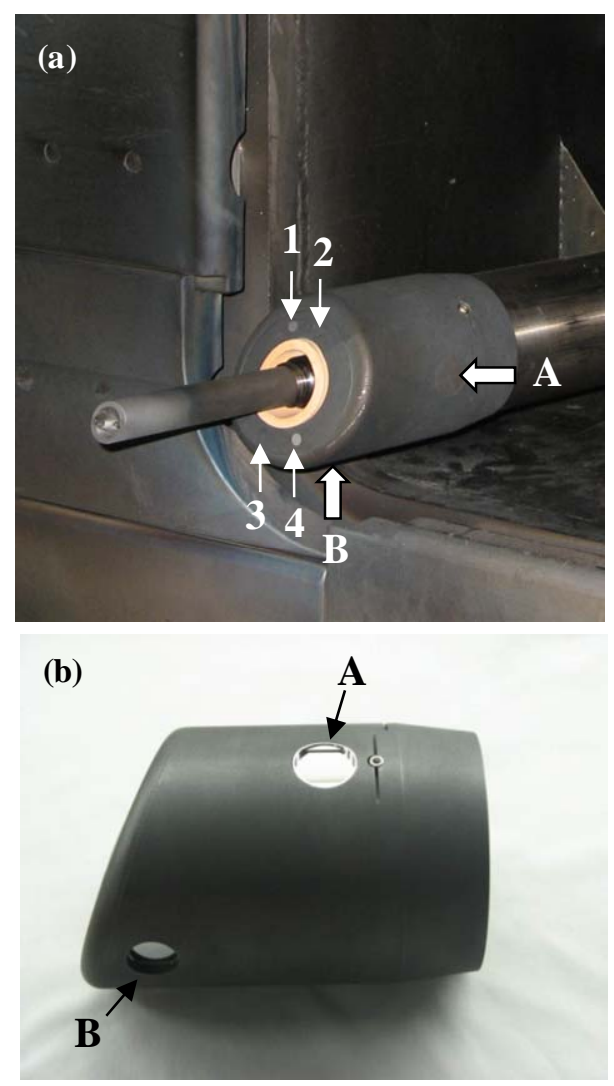

Fig. 1, D.L. Rudakov 

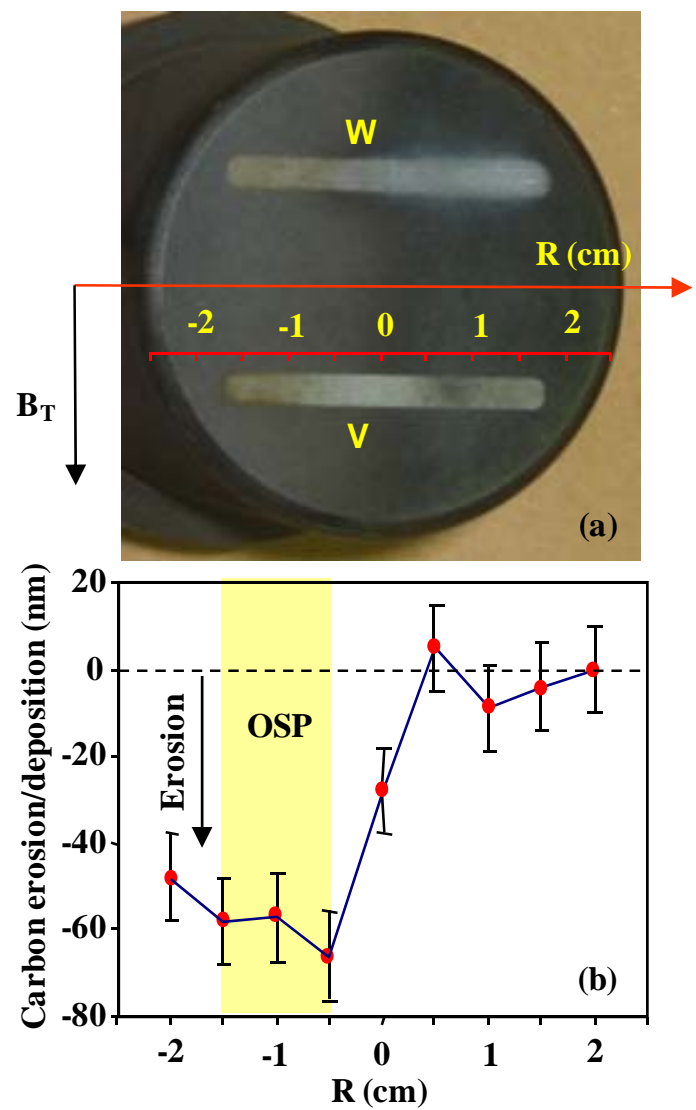

Fig. 2, D.L. Rudakov 


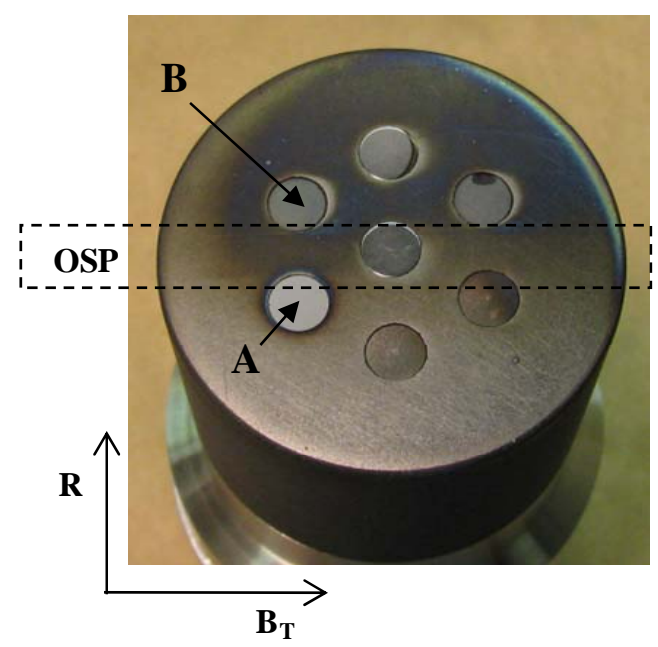

Fig. 3, D.L. Rudakov 

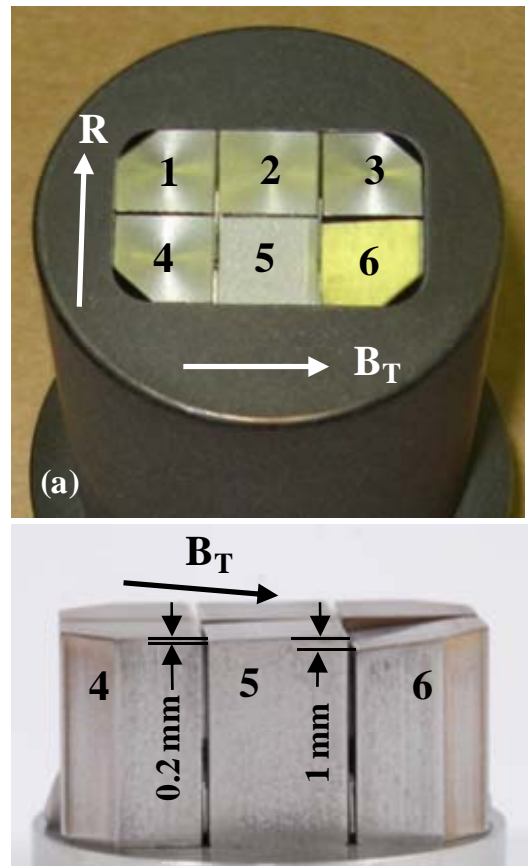

(b)

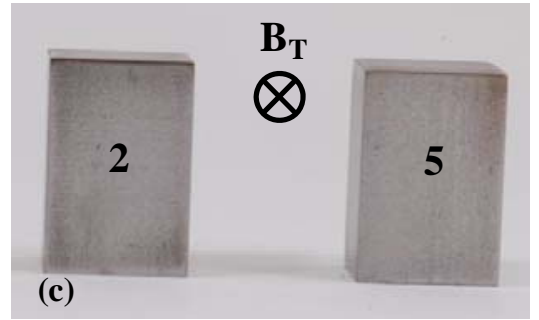

Fig. 4, D.L. Rudakov 


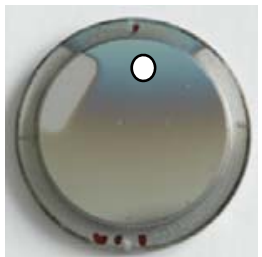

Without gas puff (a)

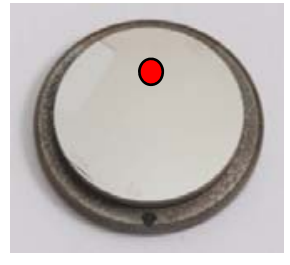

With gas puff

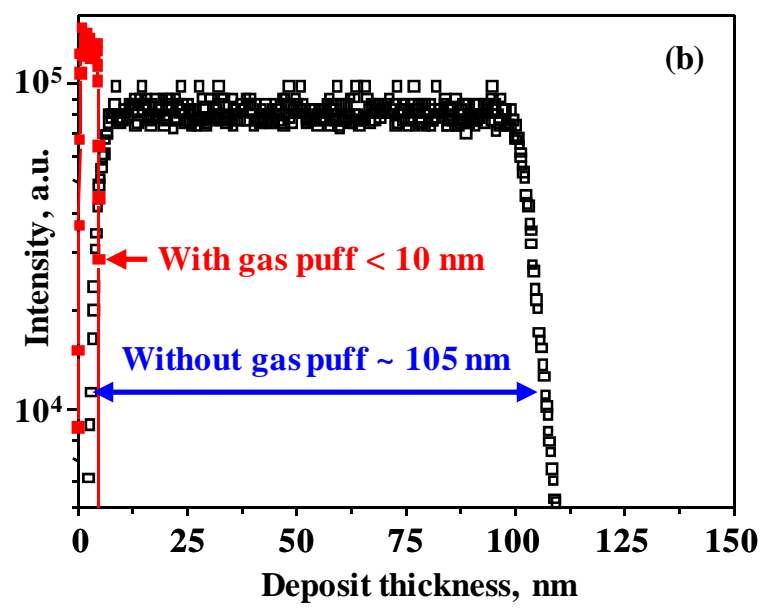

Fig. 5, D.L. Rudakov 

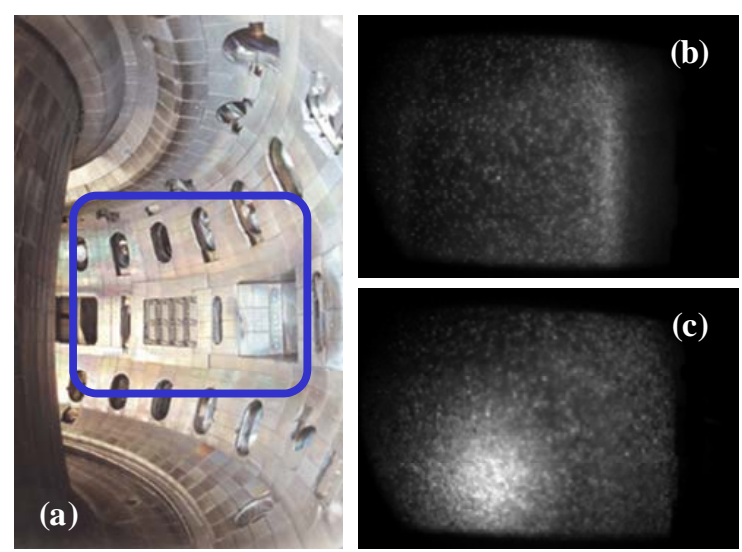

Fig. 6, D.L. Rudakov 